\title{
Appeal to Authors and Readers of the Journal Geomagnetism and Aeronomy
}

DOI: $10.1134 / \mathrm{S} 0016793210030187$

\section{Dear Colleagues!}

The first issue of our journal is dated as long as January 1961 . Thus, we will soon celebrate the 50th anniversary of our journal. The birth of our journal is related to the accomplishment of the large-scale program of the International Geophysical Year (IGY), during which a large volume of experimental data was obtained in the course of the ground-based, rocket, and balloon studies. The epoch of space-borne experiments was made during IGY. An analysis of these data opened a new page in understanding the physical processes responsible for the development of geomagnetic and ionospheric disturbances. The volume and significance of reached results were so considerable that they could not be published in the scientific journals that existed in our country. Therefore, it became necessary to create a new journal. Yurii Dmitrievich Kalinin, deputy director of the Institute of Terrestrial Magnetism, Ionosphere, and Radiowave Propagation, Russian Academy of Sciences, became an initiator and founder of this journal. At that time, the name of the journal Geomagnetism and Aeronomy naturally reflected our knowledge of physical phenomena in the near-Earth space, resulting in the generation of geomagnetic auroral disturbances and the stimulation of the physicochemical processes that reflect the unified process originating on the Sun and in the heliosphere and subsequently proceeding in the magnetosphere, ionosphere and upper and middle atmosphere. Obtained data also indicated that solar activity variations affect climatic parameters.

As our knowledge of the physical processes in the near-Earth space and heliosphere deepen, the subjectmatter of the papers published in our journal became also more extensive. At present, this subject-matter includes a wide range of issues in the field of solar-terrestrial coupling: physics of the interplanetary space, cosmic rays, solar wind, interaction between the solar wind and magnetosphere, physical processes in the magnetosphere and ionosphere, magnetic storms and substorms, physicochemical processes in the upper and middle atmosphere, effect of solar variability and activity on processes in the atmosphere and climate, main geomagnetic field and its secular variations, excursions and inversions, and active impact on ionospheric and magnetospheric processes. However, up to the present, the list of these issues did not include one of the key links, namely: physics of the Sun. It is absolutely evident that most listed problems are anyhow related to physical processes developing on the Sun or have the nature similar to the nature of these processes. The latter can be illustrated by the generation of the main geomagnetic field. Physical processes on the Sun and in the interplanetary medium are in many respects responsible for the subsequent development of events in the chain of the solar-terrestrial coupling, including magnetospheric-ionospheric processes. Therefore, the editorial board of the journal decided to widen the subject-matter of published papers, having included studying physical processes on the Sun. Thus, the subject-matter of the journal Geomagnetism and Aeronomy will include the entire complex of problems of solar-terrestrial coupling.

In this context the name of the journal Geomagnetism and Aeronomy became slightly out of date and does not reflect the variety of the problems considered in the journal. However, it is almost impossible to change the name of the journal because this will disturb the historical succession of publications. At the same time, it is reasonable to add the headline "Problems of Solar-Terrestrial Physics" to the cover page in order to reflect all problems considered in publications. On the proposal of the editorial board, such a subtitle was introduced in 2010.

The editorial board of the journal always tried to maintain a high scientific level of its publications. For the last five years, the average impact factor of Geomangetism and Aeronomy has been 0.445, which is a high value for Russian scientific journals. In 2007 the impact factor of the journal reached 0.947; as a result, the journal is among the best ten journals of the Russian Academy of Sciences according to this index.

Since the journal anniversary approaches, the editorial board asks the authors and readers of the journal to send not only original papers but also reviews on individual scientific problems in the scope of the journal subject-matter, including physics of processes on the Sun. 